\title{
GESTIÓN CULTURAL COMO MEDIACIÓN \\ PEDAGÓGICA EN LOS PROCESOS \\ ETNOEDUCATIVOS: CASO INSTITUCIÓN \\ EDUCATIVA ANTONIA SANTOS, CARTAGENA DE \\ INDIAS, COLOMBIA
}

\author{
Teresa Cassiani Herrera ${ }^{1}$ \\ Denice Casseres Gómez ${ }^{2}$ \\ Martha Herrera Valdés ${ }^{3}$
}

\begin{abstract}
Resumen
El ejercicio de revisión de la Gestión Cultural como Mediación Pedagógica en Colombia, pasa por hacer un recorrido a la historia de sus comunidades negras, afros, raizal y palanquera, es mirar el fenómeno de diáspora africana ${ }^{4}$, el movimiento social afrocolombiano y la visión de gestión desde sus prácticas étnicos-culturales. Descubrir cómo los negros y negras traídas desde áfrica y sometidos a la esclavitud, lucharon por su libertad y resistieron para salvaguardar su cultura e identidad, se traduce en un ejemplo de gestión cultural donde, además de proteger su herencia étnica, recrearon los saberes propios y adaptaron
\end{abstract}

\footnotetext{
1 Palenquera, docente etnoeducativa, coordinadora IE Antonia Santos. LC en Ciencias Sociales y económicas (U de C Atlántico), Esp. Administración, Maestría en Genero, identidad y ciudadanía intercultural (U Uraccan, Nicaragua, ex consultiva de comunidades negras. Semillero grupo RUECA. Correos: teremalc2@hotmail.com, teremalc28yahoo.com

2 Docente Etnoeducativa en la Institución Educativa Antonia Santos. Licenciada en Ciencias Sociales. Especialista en formación: Metodología de la lúdica. Responsable del Periódico Estudiantil; Noti- etno la escuela es una y para todos. Correos: dcasseres@hotmail.com

3 Magister en Educación. Lic. educación Religiosa. Docente invitada Maestría Universidad De Cartagena, Tutora del Programa Todos a Aprender (MEN), docente invitada diplomado Universidad Tecnológica de Bolívar, Semillero del grupo RUECA. Correo: marthaligia15@gmail.com 4 Exilio forzado de millones de africanos para ser sometidos a una esclavitud brutal (Cartilla n5 (2015).
} 
nuevos a partir de las propias realidades territoriales, culturales y económicas de sus comunidades. Proceso que se refleja, en parte, con el reconocimiento y garantía del Estado a la educación para grupo étnicos o etnoeducación, definida en la Ley general de educación o ley 115 de 1994. Para el desarrollo de este trabajo se utilizó una metodología cualitativa, con el sello particular de lo étnico cultural, ya que las protagonistas son sujetos y objetos de la investigación. La experiencia etnoeducativa para el caso de la Institución Educativa Antonia Santos del Distrito de Cartagena, se constituye en un modelo de valores desde lo afro, condición que marca la diferencia frente otras instituciones. En este proceso se recurre a la gestión cultural como mediación pedagógica desde el nivel preescolar y se convierte en una de la reconquista realizada por las comunidades negras para el fortalecimiento de la identidad; con una visión propia y diferente de aprender, proporcionándole un sello particular a la educación para los grupos étnicos.

Palabras clave: Gestión cultural, identidad, diáspora, etnoeducacion y libertad

\begin{abstract}
The review exercise of Cultural Management as a Pedagogical Mediation in Colombia, goes through a tour of the history of its black communities, Afros, raizal and palanquera, is to look at the phenomenon of the forced exile of millions of Africans to be subjected to slavery Brutal best known with the term African Diaspora, 1 the Afro-Colombian social movement and the vision of management from their ethnic-cultural practices. Discover how black people brought from Africa and subjected to slavery, fought for their freedom and resisted to safeguard
\end{abstract}


their culture and identity, translate into an example of cultural management where, in addition to protecting their ethnic heritage, recreated their own knowledge and Adapted new ones from the own territorial, cultural and economic realities of their communities. This process is reflected, in part, with the recognition and guarantee of the State to the education for ethnic groups or ethnoeducation, defined in the General Education Act or Law 115 of 1994. For the development of this work a qualitative methodology was used, with the particular stamp of the cultural ethnic, since the protagonists are subjects and objects of the investigation. The ethnoeducative experience for the case of the Educational Institution Antonia Santos of the District of Cartagena, constitutes a model of values from afro, a condition that makes the difference in front of other institutions. In this process, cultural management is used as pedagogical mediation from the pre-school level and becomes one of the reconquest carried out by the black communities for the strengthening of identity; With a vision of their own and different from learning, providing a particular seal to education for ethnic groups.

Keywords: Cultural management, identity, diaspora, ethnoeducation and freedom 


\section{Introducción}

Abordar el proceso actual de etnoeducación con enfoque afro y específicamente la estrategia de gestión cultural como mediación pedagógica, nos insta a la búsqueda de la intersección etnia/gestión y cultura. Para ello es necesario: primero, un recuento de la historia de los pueblos raptados desde África y sometidos a la esclavitud durante la colonia en Colombia, del cómo persiste la discriminación, y ante todo cuales han sido las luchas y resistencia por los derechos a la libertad, identidad y autonomía de las comunidades palenqueras, raizales y afrodescendientes.

Seguidamente se describe los procesos etnoeducativos con enfoque afro que se fortalecen con las garantías expresadas en el marco de la Constitución Política de Colombia de 1991, La Ley de comunidades negras y la Ley General de educación.

Finalmente se presenta la experiencia de la Institución Educativa Antonia Santos del Distrito de Cartagena, con sus logros y dificultades.

\section{Antecedentes}

\section{La Gestión Cultural para la Liberación desde la Diáspora Afroco- lombiana y Movimiento Social Afro}

La diáspora africana hacia América acaecida por la aberrante práctica del esclavismo por los europeos en el periodo colonial, suscito diversos procesos de liberación en los pueblos negros subyugados, parafraseando a Cassiani (2014, p. 116) estas formas de resistencia confluyeron en un solo movimiento generalizado de rebeldía e inconformidad por la inhumana condición de esclavización, generando 
además de serios problemas para la sociedad colonial, situaciones de inestabilidad al sistema mismo.

Hombres y mujeres que lucharon por reconquistar su libertad, que resistieron para conservar sus identidades, lenguas y costumbres. Y fue en La Nueva Granada, hoy Colombia, que germinó el primer tratado de paz producto de un proceso de gestión étnico-cultural y político por los esclavizados y las esclavizadas, así lo describe Navarrete y cols.:

A comienzo del siglo XVII, durante el gobierno de Don Gerónimo de Suazo y Casasola de la ciudad y provincia de Cartagena, los cimarrones del Palenque de la Matuna, liderados por el rey del arcabuco, Domingo Bioho, fueron atacados por las fuerzas del orden en varias ocasiones. El gobernador, considerando las complicaciones que se tendrían para acabar con ellos y en vista de que los cimarrones que sobrevivieron al asedio de las milicias desistieron de un levantamiento, prefirió negociar la paz... Los gastos de alistamientos de las escuadras y la dificultad para exterminar a los pobladores de la Matuna llevaron al gobernador a diligenciar un tratado de paz con ellos. (2014, p. 41).

Pero no solo se trató de confrontación armada contra quienes se irrogaban un supuesto derecho de propiedad a la vida y cuerpos, no, también consistió en reconquistar y proteger todo aquello que les permitía ser personas e identificarse como pueblo, así lo evidencian las investigaciones relacionada con la Diáspora Africana en América (Friedman, Múnera, Navarrete, Hernández, Cassiani) y muchos coinciden en que las estrategias utilizadas los esclavizados fueron diversas y conllevaban al mismo objetivo Ser libre, formar una nación libre similar a su territorio africano, entonces, que significaba ser libre para los ancestros/as africanas/os. 
Poseer un territorio ${ }^{5}$, tener libertad de ejercer el derecho a ser negros, negras y africanos, es decir, a practicar su identidad cultural, sus prácticas tradicionales y sus costumbres; por ello es importante revisar los conceptos al respecto.

Para Michel Agier (2002, p. 295) "La identidad cultural interviene como un argumento en la reivindicación y la lucha por derechos sociales y políticos de las poblaciones históricamente".

Según Hernández en el libro Genealogía de la Identidad cultural palenquera (2014, p. 56), cuando se habla de Identidad cultural se debe entender, fundamentalmente, una dirección análoga de la existencia histórica de los individuos y de los pueblos, por cuanto ella supone un ser que se afirma a sí mismo en la existencia, pero que no se concibe acabado y, se proyecta como queriendo ser sin aniquilarse, si no transformándose en forma permanente.

Y es que la identidad cultural es una construcción colectiva, en donde cada individuo juega un determinado papel, podemos decir; que las actuales identidades de los Afrocolombianos, los negros/as, los palenqueros/as se constituyó en gran parte en el trasegar de la Diáspora africana, Lo que significa que los elementos culturales traídos de áfrica, se recrearon, ejemplo de ellos es la lengua criolla palenquera, que fue uno de los resultados de la estrategia que utilizaron los esclavizados para entenderse con los otros y otras de su misma condición, y que tiene una importancia relevante en las identidades de los pueblos étnicos, como lo plantea Rutsely Simarra (2014, p. 76):

Uno de los recursos más valiosos y representativos del patrimonio cultural de una comunidad lo constituye

5 "El territorio es entendido como espacio vital en el que habitamos y nos desarrollamos, asociado al control de la propiedad; aspectos estos de los que dependen el ejercicio de la autonomía de la comunidad" (Caicedo, 2000 citado por Cassiani, Archipiz y Umaña, 2002, p.21). 
su lengua, por cuanto a través de ella, se transmite y se aprende la cultura misma, se difunde la cosmovisión, los principios, los valores del colectivo, las normas de convivencia.

En el caso de la lengua criolla palenquera tiene su base en la lengua de la familia Bantú, la esencia de la identidad africana prevalece.

Hoy esta lengua propia junto a otros elementos étnicos culturales como la forma de vestir y los peinados, son los principales motivos de discriminación contra los y las negras en el departamento de Bolívar y Cartagena. Esos mismos elementos llevaron a que los palenqueros y las palenqueras se sintieran sensibilizados y motivados para iniciar un proceso de gestión de fortalecimiento de la identidad.

Fueron ellas, las mujeres vendedoras de frutas, dulces y víveres de Palenque en Cartagena, que iniciaron el proceso de gestión en la defensa de sus derechos al ser mujer palenquera; Pascual Miranda y Nao Casseres narran (en entrevista a Miguel Obeso, Palenque, febrero de 2017) que entre las primeras mujeres de Palenque que salen a Cartagena estaban Julia Cassiani, (tía Chalo), Mauricia, Inés Cassiani, Máxima Salgado, tía Caña, Pila Valdés. Igualmente, la señora Cristina Salgado cuenta (en entrevista a Teresa Cassiani, Cartagena, 2015) que cuando apena era una niña de aproximadamente 13 a 14 años, enfrentaron a policías inescrupulosos que querían aprovecharse de las mujeres palenqueras exigiendo el pago del impuesto por vender en las calles de Cartagena.

Es importante destacar que el quehacer del pueblo palenquero está inmerso en los momentos que define Hernández (2014. p. 82) como parte de la lucha del pueblo afro en general y que sintetiza en cuatros momentos así: 
1. Durante los tiempos de la esclavización y colonización... mediantes construcción de Palenques, kuagros, cabildos y cofradías;

2. Últimas décadas del siglo XIX, y su participación activa en los episodios sociales y comunitarios de la primera mitad del siglo XX, y su expresión concreta se manifiesta en la inmersa labor de numerosos intelectuales y los intentos de articulación nacional como expresión del segundo momento transicional;

3. Trabajo comunitario, movimiento social afrocaibeño y poder como referente para la articulación, consolidación y proyección nacional en el tercer momento que surge el calor de la crisis de los años 80 e inicio de los procesos de formalización nacional y regional de los derechos legales constitucionales; y

4. Movimiento social y formas organizativas, retos y desafíos actuales en el contexto del capitalismo globalizado.

Con el objetivo de cultivar, fortalecer y recrear los elementos de la identidad cultural palenquera, el movimiento social palenquero presenta la propuesta ante la Unesco para declarar a Palenque patrimonio intangible e inmaterial de la humanidad, y que fue un ejercicio maravilloso de admirar, e imitar; el cuál se caracterizó por:

- La planeación, el desarrollo de trabajo colectivo, las permanentes reuniones en la comunidad para analizar el objetivo y alrededor ese gran objetivo el trabajo por mesa; luego trabajo de revisión del material (con un equipo más reducido en cantidad de personas).

- La gestión ante el Ministerio de Cultura, más específicamente ante Instituto Colombiano de Antropología, quien una vez 
acepta la propuesta, la presenta a la entidad internacional, en este caso a la Unesco.

La declaración de Palenque como Patrimonio Intangible de la Humanidad se constituyó en un apoyo significativo al proceso etnoeducativo, definida en el artículo 55 de la Ley 114 de 1994 como...

la que se ofrece a grupos o comunidades que integran la nacionalidad y que poseen una cultura, una lengua, unas tradiciones y unos fueros propios y autóctonos. Esta educación debe estar ligada al ambiente, al proceso productivo, al proceso social y cultural, con el debido respeto de sus creencias y tradiciones.

Lograr el reconocimiento de la etnoeducación o educación para grupo étnico, es el resultado de todo un proceso de gestión desde la perspectiva étnica, ya que las clases política tradicional de un país acepte un proceso de etnoeducación hasta cierto punto es reconocer un proceso de discriminación y racismo. Como se plantea en Derecho a no ser discriminado, del Observatorio de Discriminación Racial:

El estado colombiano ratifico el 1981 la Convención Internacional de la ONU sobre Racismo, Discriminación Racial, Xenofobia... Más de un cuarto de siglo después, los compromisos adquiridos por el Estado siguen siendo una tarea pendiente a pesar de haberse reiterado al adoptar la constitución política de 1991 y al apoyar la declaración y programa de Acción de la Conferencia Mundial de Durban sobre el tema en el 2001. (Rodríguez, Alfonso \& Cavelier, 2008, p. 13) 


\section{La Etnoeducación desde la Perspectiva Afro}

En el apartado anterior se sintetizó algunos momentos históricos relevantes que permiten identificar el recorrido y esencia de los procesos de gestión cultural de la comunidad afro y que fortalecen la etnoeducación en Colombia.

Sin embargo, los procesos etnoeductivos que, como se advirtió, son resultado de la lucha del pueblo negro, afro, raizal y palenquero por el derecho a tener identidad propia, a cultivarla sin que ello constituya un impedimento para el desarrollo comunitario y personal, hacen parte del cumulo de reconquista que se concretó formalmente en la Constitución de 1991 y la Ley 70 de 1993 -ley de Comunidades Negras- en la que se reconocen una serie de derechos sociales, económicos, políticos y culturales a los descendientes africanos. Allí se crean las condiciones legales para el impulso de programas de etnoeducación acorde con las condiciones concretas de las comunidades, en el capítulo VI se define mecanismos para la protección, desarrollo de los derechos, de la identidad cultural:

Artículo 34. La educación para las comunidades negras debe tener en cuenta el medio ambiente, el proceso productivo, y toda la vida social y cultural de estas comunidades. En consecuencia, los programas curriculares aseguraran, reflejaran el respeto y fomento de su patrimonio económico, natural, cultural y social, sus valores artísticos, sus medios de expresión y sus creencias religiosas. Los currículos deben partir de la cultura de las comunidades negras para desarrollar las diferentes actividades y destrezas en los individuos y en el grupo, necesarios para desenvolverse en su medio social. 
Artículo 35. Los programas y los servicios de educación destinados por el Estado a las comunidades negras deben desarrollarse y aplicarse en cooperación con ellas, al fin de responder a sus necesidades particulares, y deben abarcar su historia, sus conocimientos y técnicas, su sistema de valores, sus formas lingüísticas y dialectales y todas las demás aspiraciones sociales, económicas y culturales.

Además, destacan la Ley 115 de 1994 -ley general de educación-, la Ley 725 de 2001 "por la cual se establece el día nacional de la Afrocolombianidad", el Decretos 804 de 1995, "por la cual se reglamenta la atención educativa para grupos étnicos"; el Decreto 1122 de 1998 reglamentario del Artículo 39 de la Ley 70 de 1993 "por la cual se expiden normas para el desarrollo de la Cátedras de Estudios Afrocolombianos, en todos los establecimientos de educación formal del país".

Esto solo para mencionar los relacionados directamente con la educación para el pueblo étnico Afrocolombiano. En este sentido se podría imaginar que en Colombia las comunidades negras, afro y raizales tienen garantizados sus derechos, no obstante, sobre el tema estudiado surgen las siguientes preguntas:

¿Cuáles son las principales razones de la implementación de la etnoeducación?

¿Cuáles son las principales barreras que impiden el pleno desarrollo de la etnoeducación?

¿Cuáles son las nuevas propuestas del pueblo afrocolombiano?

¿Cuál ha sido el real alcance de este proceso hacia las comunidades y el resto del pueblo afrocolombiano? 
Responder tales cuestionamientos implica una aproximación a la realidad y persistencia del fenómeno de discriminación y racismo, que se instituyen como los primeros detonantes para el desarrollo de la etnoeducación. Fenómenos que las comunidades negras, afros, palenqueras y raizales han sufrido desde la traída forzosa de los pobladores del continente africano hacia América.

Según el último censo realizado en Colombia se muestra claramente la discriminación y el racismo ejercido desde las estructuras del Estado así:

...el último censo de población realizado en el año 2005, en Colombia hay 41.468 .384 personas, de las cuales 4.311.757 correspondientes al $10.62 \%$ se reconocieron como, afrodescendientes, palenqueras y raizales, el 3.36\% india y el 0.1\% ROM. Además del castellano se hablan 68 lenguas pertenecientes a grupos étnicos el $60 \%$ a indígenas dos lenguas criollas afrodescendientes que son el creole, el palenquero y el romaní, Sin embargo, los movimientos sociales de comunidades afro cartagenera afirman que la población afrodescendiente, corresponde al 26\% aproximadamente de la población nacional; las mujeres son el 50.5\%. Del total de esta población, el 72\% habita en las zonas urbanas y el $28 \%$, se ubica en las zonas rurales. En "La Raza en Cifras" del Observatorio de Discriminación Racial que presenta datos y estadísticas de la población afrocartagenera, negra y palenquera en Colombia, así como el primer Informe sobre Discriminación y Derechos de la Población Afrodescendientes, evidencian que la población afrocartagenera sufre discriminación en el goce de casi todos sus derechos sociales. Por ejemplo, la esperanza de vida al nacer para los hombres afrocartageneros de 64,6 años y para las mujeres es de 66,7 años mientras que para 
el resto de los hombres no afros es de 70,3 años y de las mujeres es 77,5 años, es decir que la esperanza de vida de las mujeres afrodescendientes es menor en 11 años que la de las mestizas. La tasa de mortalidad infantil (por 1000 nacidos vivos) de las comunidades afro es para los hombres, de 48,1\% y para las mujeres de 43,9\% frente al $26,9 \%$ para los hombres y $21,0 \%$ para las mujeres del resto de la población, esto significa que la mortalidad de los niños/as afrodescendiente/as, es el doble de la del resto de la población. La incidencia del hambre en la población mestiza es de $6,11 \%$, mientras que en los afrocartageneros es del 14,30\%, prácticamente el doble" (Asociación Graciela Cha Inés, 2010, p. 31).

Estas cifras, si bien se limitan a la población afro cartagenera, son similares o incluso más preocupantes en el resto de los territorios afrocolombianos.

Respecto a la educación impartida a las comunidades afros, era homogeinizante; lo que conllevaba al debilitamiento de su identidad, a la separación entre la escuela y la comunidad, docente y comunidad, pero además le quita la autonomia a las comunidades de ser objeto, sujeto, fuente y método de sus propios procesos investigativos y desarrollar pedagogía acorde a las realidades de la comunidad. Situación que describe Hernández (2012):

Los procesos educativos formales, que en ocasiones algunos van cargados de discriminación y de imposición de modelos de vida ajenos al de la comunidad, han sido una de las principales causas de un progresivo proceso de deterioro de los ejes de identidad, por lo cual la comunidad se ha tenido que adecuar sus propias estrategias de sobrevivencia cultural; es así como, en la década de los ochenta, se levanta 
y organiza alrededor de esta problemática para, después de largos procesos de reflexión, elaborar una propuesta de innovación educativa (Educación propia o Etnoeducación) que realmente reconozca, respete y potencie el desarrollo de los valores históricos, sociales y culturales de esta comunidad afrocolombiana.

De allí, que la comunidad toma la decisión autónoma de desarrollar procesos propios y por ello siente la necesidad de revisar los temas investigados y escritos por los foráneos ${ }^{6}$ quienes le imprimían su subjetividad a la investigación en detrimentos de los derechos y de la cosmovisión de las comunidades; realizar procesos de investigación, de estudiar, analizar y sensibilizar a las nuevas generaciones sobre su realidad presente y futuro (proyección). Iniciando un proceso de sensibilización a aquellas comunidades en sus derechos ancestrales: colectivos consuetudinarios $^{7}$ que mayor debilidad presentaban de la cultura, de la historia, de su cosmovisión, de su lengua, de su religiosidad. De esta forma se empieza a pensar en el proyecto comunitario etnoeducativo, esto en correspondencia con el origen de la etnoeducacion que surge de los pueblos y comunidades que mantienen viva su cultura, su identidad, por ello es importante resaltar que la etnoeducacion no pretende solucionar conflictos económicos estructurales de la comunidad, da herramientas para la proyección de los pueblos afrodescendientes, en la medida que este asume la Indagación acerca de cómo era, cómo es y cómo se proyecta la comunidad en términos de debilidades y fortalezas, se decantan de consenso, prioridades, o problemas más sentidos, y se procede a elaborar la visión y misión, con el respectivo plan de gestión. Es una iniciativa comunitaria, con la que se pretende construir valores, fortalecer la cohesión social, reafirmar y reconstruir identidad personal y colectiva.

6 Términos que hace referencia a las personas que no son propia de un territorio.

7 Son los derechos que tienen los pueblos de ser protegidos. 
Dentro de los aspectos a revisar y analizar del plan global de vida comunitario se prioriza lo histórico, político, organizativo, territorio-ambiental, social, familiar, religioso educación, vivienda, salud tecnologías, técnicas, económico deporte, lúdica y arte. En correspondencia con ellos se define el tipo de hombre y de mujer que la institución pretender formar debe corresponder a los perfiles de persona y sociedad que el pueblo desea construir a partir de lo explicitado en el proyecto de vida; se establecen perfiles para estudiantes, docentes, administrativos, comunidad y establecimiento educativo.

En este sentido, el proyecto global de vida se asume como una propuesta de etnodesarrollo, que tiene como base la práctica del umbutu $^{8}$, dado que la comunidad es quien determina su propio destino desde sus referentes sociales, históricos, étnicos y culturales desde un trabajo colectivo, donde priman las necesidades colectivas.

Entonces se puede definir la etnoeducación como una herramienta pedagógica-comunitaria que permite un buen entendimiento entre la escuela/comunidad y entre el docente/comunidad, asimismo permite el vínculo directo comunidad/escuela que posibilita que desde la etnoeducación se aporte al proyecto global de vida de la comunidad

Para Hernández (2012) se destacan tres momentos y estadios de la etnoeducación:

Consideramos que la Etnoeducación a lo largo y ancho de su existencia ha atravesado por tres grandes momentos en los cuales adquirió una connotación y rasgos específicos que definieron el derrotero de esta a

8 La actitud mental prevaleciente entre los nativos del extremo sur de África, surge del dicho popular "umuntu, nigumuntu, nagamuntu", que en idioma zulú|zulú significa "una persona es una persona a causa de los demás." Hay varias traducciones posibles del término al español, las comunes son: "Humanidad hacia otros" "Si todos ganan, tú ganas" "Soy porque nosotros somos" "Una persona se hace humana a través de las otras personas". 
lo largo de las tres décadas de duración. Primer momento: de genesis y arranque. Segundo Momento: La Aprobación de la Ley 70 de 1993 y el soporte Legal. Tercer Momento: Los inicios de Diplomados y el surgimiento del Instituto de Educación e Investigación Manuel Zapata Olivella y la Asociación de Etnoeducadores de Comunidades Afro caribeñas.

Este proceso fue impulsado por la comunidad, en donde trabajaban abuelos, abuelas, tío, tía, Jóvenes, hombres y mujeres sin distinción, se unieron en pro del desarrollo del fortalecimiento, aquí se desvela la gestión comunitaria.

\section{Resultados de la Investigación}

\section{Barreras de la Etnoeducación}

En dialogo con Teresa Cassiani, Una de las barreras más importantes fue de orden administrativo, pues los funcionarios no tenían conocimientos de la etnoeducación al respeto, a pesar de las normatividades y leyes expedidas, si los gobiernos de turno no asumían el cumplimiento de las normas quedaba a la voluntad política de los funcionarios locales o simplemente los que se identificaban con los planteamientos. En ese mismo sentido fue el de los funcionarios que concluyeron que la etnoeducación le restaba la posibilidad de tener cuotas burocráticas con su trabajo, esto debido a que en muchas zonas, municipios y territorio de comunidades negras como fue el caso de Palenque de San Basilio donde las primeras personas nombradas como docentes con un contrato de provisionalidad tenían el perfil que la comunidad requería para la enseñanza de la cultura, lengua y tradiciones, no obstante carecían del título requerido (licenciatura) según el Ministerio de Educación Nacional. Se le otorgo nombramiento a tres 
docentes para la enseñanza de la lengua criolla palenquera (Bernardino Pérez, Sebastián Salgado y María Los santos Reyes), y posteriormente le permitieron mediante dos años de prorroga profesionalizarse, (Entrevista realizada en Palenque, por Martha Herrera y Denice Casseres a Teresa, febrero del 2017).

Es importante destacar que la implementación de la etnoeducación en cada territorio fue diversa, de acuerdo a las necesidades, y a la sensibilización de la comunidad. En el caso de Palenque de San Basilio; es la comunidad del Caribe colombiano donde se da inicio a este proceso con las siguientes estrategias:

- Jornadas de investigación con la comunidad y para la comunidad

- Puesta en escena de lo investigado

- Desarrollo de talleres con la comunidad

- Jornada pedagógica con los docentes, estudiantes y administrativos en la comunidad

- Encuentros y desencuentros con investigadores

- Sistematización de experiencia y del método de la consulta a la memoria colectiva

- Presentación a la Unesco la propuesta de Palenque Patrimonio Intangible de la Humanidad

- Elaboración, concertación e implementación del Plan de salvaguarda para Palenque 
- Planteamiento del Modelo Pedagógico para la comunidad Palenquera.

Además de las dificultades mencionadas, agrega Hernández (2012) que los avances del programa de Etnoeducación fue afectado por una serie de problemáticas que en su orden son las siguientes:

- Crisis de fundamentación teórica

- Calidad de los docentes y /o recursos académicos.

- De orden administrativo y de proyección a la comunidad

- Carencia de recursos logísticos y económicos.

A pesar de ello, las instituciones educativas han continuado algunos programas y proyecto que en diferentes niveles intentan romper las barreras.

En ese orden de ideas en Cartagena la Etnoeducación se inicia con el despertar de los y las Palenqueras que se trasladaron de la comunidad hacia la ciudad y hace parte del primer momento de génesis y arranque que Hernández (2012, p. 5) plantea:

Desde el año 1979 se viene desarrollando...en los núcleos palenqueros ubicados en Cartagena, una serie de actividades educativas cuya finalidad es fortalecer la identidad étnica-cultural, elevar su nivel de conciencia étnica, lograr la sensibilización de diferentes grupos sociales, con los cuales interactúa, de tal manera que conozcan lo que representa la cultura de ese grupo étnico en la nación colombiana. 
El centro de todas esas actividades fue el Barrio de Nariño9, específicamente la escuela San Luis Gonzaga; era el único sitio que había en la comunidad donde se podría desarrollar actividades comunitarias, así, que fue el epicentro donde se inicia el proceso etnoeducativo en la ciudad de Cartagena. Esta experiencia esta sistematizada en el proyecto de lengua criolla Minino a Chitia ku ma kombilesa suto ${ }^{10}$ :

\section{Inicio de la Etnoeducación en Cartagena}

La Institución Educativa Antonia Santos, sede San Luís Gonzaga se encuentra ubicada en el barrio Nariño, atiende a una población afro descendiente. Funciona desde el año de 1944 en poder de los señores Jesuitas, posteriormente fue comprado por los padres Claverianos en 1954.

Es importante destacar que a principios de las décadas de los años ochenta (80), el "renacimiento" del movimiento afro en la ciudad de Cartagena, tuvo sus primeros cimientos en la sede de la escuela San Luís Gonzaga, la juventud palenquera que vivían en el sector utilizaban la escuela para alfabetizar a la población de Palenque que allí vivía, en especial a las mujeres. La escuela de San Luís fue el escenario propicio para las reuniones donde se hablaba, estudiaba, discutía, y se recreaba la cultura palenquera y afrodescendientes en la ciudad de Cartagena de Indias.

Desde el año 1996, el equipo de Etnoeducación Bolívar (la mayoría Palenquero/as), detectaron la necesidad de desarrollar programa de fortalecimiento de la identidad en algunas instituciones de la ciudad de Cartagena, donde la mayoría eran palenquer@s y/o afrodescendientes,

9 Sector de la ciudad de Cartagena de Indias, donde habitaban gran mayoría de la población Palenquera.

10 Dialoguemos entre amigos nuestra lengua 
y con una visión amplia de la realidad en la ciudad, se inicia el trabajo de Etnoeducación, con cuatro docentes voluntarias; como consecuencia de ello en el año 1999 su Proyecto Educativo Institucional (PEI) fue premiado por el Ministerio de Educación Nacional reconocido como sobresaliente por desarrollar su proyecto etnoeducativo con énfasis en el fortalecimiento de las costumbres y tradiciones.

La Institución Educativa Manzanillo del Mar y la escuela de lengua criolla palenquera Minino a Chitia Ku Suto, de la sede San Luis Gonzaga de Antonia Santos, fueron tomadas como ejemplos de colegios que lideran en la ciudad, dinámicas pedagógicas etnolingüísticas. (Ministerio de educación Nacional, 2010).

La propuesta desarrollada en San Luis tiene como norte la cultura Palenquera, por lo que simbólica, cultural e históricamente representa para las comunidades afrodescendientes, para Colombia, América y el mundo.

En el año 2002 la escuela San Luís Gonzaga fue fusionada a la Institución Educativa Antonia Santos; el proceso etnoeducativo en la institución ha continuado con grandes éxitos y retos; ser escuela Etnoeducativa implica conocernos como actores de un país con una diversidad cultural expresada en multiplicidad de identidades y expresiones culturales de pueblos y comunidades que conforman la nacionalidad.

Es así como la Etnoeducación se ha tomado por lo menos a 21 escuelas Cartageneras ya que en este contexto se abren espacios legales para que la escuela se reconozca y actué en concordancia con la realidad nacional; estas instituciones son reconocidas por el decreto 0670 del 20 de mayo 2013 por el cual se determina las instituciones educativas del distrito de Cartagena donde se focalizan acciones orientadas a la 
construcción de modelos educativos pertinentes; estas se enfocan en los procesos académicos que se enmarcan en ellas y se procura mantener el legado en las actividades culturales, sociales y académicas que se ejecutan.

En el caso de la Institución Educativa Antonia Santos reconocida en Cartagena como Etnoeducativa con énfasis en la identidad del negro, existe el reto de sostener un proceso educativo que haga propio el conocimiento en todas las dimensiones de la gestión escolar, el fortalecimiento de las huellas de la cultura africana. Esta huella se hace presentes, se sienten y se expresan en el plan de estudio.

Desde la historia se ha reconocido a África como cuna en el desarrollo de la humanidad, ya que se construye en los estudiantes una imagen de África como tierra con significado de origen, en ciencias naturales se estudia el genoma humano en donde se mira las etnias, técnicas de siembra para conservar especies autóctonas, en arte se trabaja la teoría del color y la simetría en las historia en los barrios, en lengua de señas se estudia los géneros narrativos con historias contadas desde los abuelos, en religión se observan las creencias, la religiosidad propia, en humanidades se ve el contraste de poesía clásica y popular en el contexto de la cultura por medio de la valoración artística y poética de los pueblos negros, en ciencias sociales se estudia la visión del mundo afro, la gastronomía afrocaribeña, además de la danza y el folclore en la cadencia del negro y en los vestuarios autóctonos la estética en las comunidades negra, palenquera y raizal, se fortalece la lengua palenquera en la sede de primaria, siguiendo con inglés debido a que este idioma es muy importante en la vida cotidiana pues se realizan procesos de elaboración y mercadeo en dulces tradicionales y finalmente en las matemáticas se trata de articular todos los espacios escolares con la valoración de la identidad además de series, líneas poligonales, sucesiones y círculos se desarrollan a través de los peinados africanos. 
Apropiándose de los conocimientos universales que resinifiquen los saberes ancestrales de la identidad negra.

Para terminar, se resumen algunos de los logros de la etnoeducación en Cartagena:

- La expedición del Acuerdo 015 del 2 de agosto de 2004, por medio del cual el distrito de Cartagena adopta como política educativa permanente, para fortalecer y reafirmar la identidad étnica y cultural en las Instituciones Educativas Oficiales y Establecimientos Educativos Privados, el programa de Etnoeducación y la Cátedra de Estudios Afrocolombianos

- La expedición de la Secretaria de Educación, de la Resolución 019 de 2004, que crea e institucionaliza la Red de docentes etnoeducadores del distrito de Cartagena

- La vinculación formal de más de 300 educadores nombrados como directivos y docentes etnoeducadores para las instituciones educativas oficiales, esto en virtud a la Circular MEN 025 de 2004, El Decreto MEN 140 de 2006 y el Decreto Distrital 670 de 2013 que fijaron y orientaron los criterios para estas vinculaciones.

- Entre el año 2005 al 2015, la capacitación y formación de más 2000 educadores en etnoeducación, interculturalidad, diversidad étnica y cultural y en lineamientos curriculares para la implementación de la cátedra de estudios afrocolombianos tal como lo establece el Decreto MEN 1122 de 1998.

- A partir del 2014, 26 instituciones focalizadas como etnoeducativas en procesos de resignificación con acciones 
orientadas a la construcción de modelos pedagógicos que permitan incluir en su Proyecto Educativo Institucional, saberes, estrategias, metodologías que garanticen a las comunidades negras, afrocolombianas, raizales y palenqueras el derecho a un proceso educativo acorde con sus necesidades y aspiraciones en concordancia con su contexto socio-culturales

- Activa y efectiva participación en los procesos de fortalecimiento identitario, que ha permitido que en el Censo DANE 2005, en Cartagena más de 330.000 personas esto el 36\% de su población censada oficialmente, se auto reconoció como negro, afro, raizal o palenquera (Rodríguez, 2016).

\section{Conclusión}

De acuerdo con lo anteriormente expuesto, es posible afirmar que la población étnica le correspondió librar una fuerte lucha para que sus particularidades históricas, sociales y culturales fuesen reconocidas y tenidas en cuenta cómo parte importante de la sociedad. Lograr que desde la Ley General de Educación (1994) se reconociera la Etnoeducación con sus características particulares y unas orientaciones hacia la preservación de toda una herencia legada por los negros y negras esclavizadas da cuenta de la gran lucha que ha tenido este grupo étnico por pervivir a través de la historia.

La discriminación expresada en diversas formas ha sido una de la causa del proceso de gestión de las comunidades para la defensa de su cultura, lo que le ha permitido ser protagonista activa en los avances logrados ante el Estado colombiano a nivel legislativo; además de fortalecer su autonomía como pueblo negro. 
Resulta oportuno afirmar que la lucha dio frutos y que al día de hoy la cultura Afro ya es reconocida y más respetada en esta sociedad, aunque todavía existen tensiones propias de proyecto y programa de innovación. Sin embargo, lo que se ha ganado ha sido también gracias al movimiento social afrocolombianos que jugó un papel trascendental en la conquista de la etnoeducación.

No obstante, las etnoeducación tuvo que superar a algunas barreras, como por ejemplo el desconocimiento de algunos funcionarios acerca de su aplicabilidad y normatividad.

La lengua criolla palenquera se convirtió en una de la estrategia más importante para desarrollar la etnoeducacion y preservar el patrimonio cultural, dando como resultado la gestión para declarar a Palenque de San Basilio como Patrimonio Intangible de la Humanidad, suceso fundamental para salvaguarda de la identidad étnico-cultural de Palenque para Colombia y el mundo.

El reconocimiento de la etnoeducación es el resultado de un proceso de gestión desde las comunidades étnicas como aporte a la educación en Colombia. Siendo esta una herramienta pedagógica comunitaria que permite el desarrollo de los pueblos de acuerdo a sus realidades, necesidades y expectativas.

Hoy las instituciones etnoeducativas tienen un reto y compromiso con el pueblos afro, negro, palenquero y raizal; pues el movimiento social afrocolombiano le suministro las herramientas para transformar la educación y acercarla a las realidades de las comunidades, la lucha dada por las comunidades llevo a que el Estado colombiano asumiera la etnoeducación como parte de la política pública; queda en mano de las instituciones, de los etnoeducadores y del Movimiento continuar el trabajo como un solo cuerpo que dirigen sus esfuerzos a un mismo objetivo. Desde esta perspectiva desde la Institución Etnoeducativa e Inclusiva Antonia Santos se viene en un proceso de 
trabajo pedagógico permanente para fortalecer el currículo etnoeducativo con ello aportar a la construcción de una Colombia más incluyente y participativa desde la diferencia de todas sus culturas.

\section{Referencias}

Agier, M. (2002). Identidad cultural, identidad ritual: una comparación entre Brasil y Colombia En Mosquera, C; Pardo, M \& Hoffmann, O. (Edit.) Afrodescendientes en las Américas, Colombia: Universidad Nacional, ICANH.

Asociación de Mujeres Afrodescendientes y del Caribe Graciela Cha Inés (2010). Investigación diagnóstica sobre las diferentes formas de violencia de género en mujeres afrocartageneras, negras y palenqueras de las localidades 1 y 2 del distrito de Cartagena (en proceso de publicación).

Blandon, M. \& Perea, R. (2015). Debates sobre Conflictos Raciales y Construcciones Afrolibertarias, Medellín: Ediciones Poder Negro.

Cassiani, A.; Achipiz, C. \&Umaña, A.(2002), Cultura y Derecho, Cali: editores FAID.

Cassiani Herrera, A. (2014). De la historia negra: símbolo, cosmovisión y resistencia. Cartagena, Colombia: Ataole - Ediciones Pluma de Mompox S.A.

Constitución Política de Colombia (1991). Bogotá: Legis, 1991.

Congreso de Colombia. Ley 70 (1993). República de Colombia: Diario Oficial No. 41.013. Recuperado a partir de http://www.alcaldiabogota.gov.co/ sisjur/normas/Normal.jsp?i=7388.

Congreso de la Colombia. Ley 115 (1994). República de Colombia: Diario Oficial No. 41.214 de Febrero 8 de 1994. Recuperado a partir de https://www.mineducacion.gov.co/1621/articles-85906_archivo_pdf. pdf

De Ávila O., Taborda S., Hernández, D., Guerrero, L., Yances, M.,\& Muñoz, E, Colección "Afrodescendientes en Cartagena: Una historia para ser contada", Cartilla n 5 (2015). Universidad de Cartagena.

Departamento Nacional de Estadística (Dane) (2005). Censo General 2005. Bogotá.

Hernandez, R. (2012). Movimientos Sociales identidad y sujetos de poder: Análisis de las prácticas pedagógicas comunitarias y su incidencia en el 
desarrollo de los pueblos afrocaribeños y otras colectividades sociales. Cartagena: Instituto de educación e Investigación Manuel Zapata Olivella.

Hernández, R. (2014). Genealogía, de la identidad cultural palenquera y su incidencia en el movimiento social afrocolombiano, Cartagena: Casa Editorial S.A.

Ministerio de Educación Nacional (abril 29 de 2010). Experiencia etnoeducativa de Cartagena es modelo en el país. Centro virtual de noticias de la educación. Recuperado el 01 de enero de 2017 a partir de http://www.mineducacion.gov.co/cvn/1665/w3-article-229419.html Ministerio de Cultura, Instituto Colombiano de Antropología e Historia (2002). Propuesta de candidatura a Palenque Patrimonio Intangibles e Inmaterial.

Ministerio de Educación Nacional, (2001). Serie lineamientos curriculares, Cátedra de Estudios Afrocolombianos. Colombia.

Ministerio del Interior y de Justicia, Dirección de Asuntos para Comunidades Negras (2008). Afrocolombianas, Raizales y Palenqueras, Compendio Legislación Afrocolombiana. Colombia.

Navarrete, M.; Lozano B.; Cassiani, A.; Reiter, B.; Obeso, K. \& Dittman, M. (2014). De La Historia Negra, Cartagena: Ediciones Pluma de Mompox S.A.

Presidencia de la República. Decreto 1122 (1998). República de Colombia: Diario Oficial No. 43.325, de 23 de junio de 1998. Recuperado a partir de http://www.urosario.edu.co/Universidad-Ciencia-Desarrollo/ ur/Fasciculos-Anteriores/Tomo-I---2006/Fasciculo-4/pdf/2006_fa04_ decreto_1122_1998/

Presidencia de la República. Decreto 140 (2006). República de Colombia. Diario Oficial 46160 de enero 23 de 2006. Recuperado a partir de http://www.alcaldiabogota.gov.co/sisjur/normas/Normal.jsp?i=18842

Rodríguez, C.; Alfonso, T. \& Cavelier, I (coord.)(2008).Derecho a no ser discriminados. Bogotá: Observatorio de discriminación racial. Recuperado el 22 de marzo del 2017 a partir de http://babel. banrepcultural.org/cdm/singleitem/collection/p17054coll12/id/1

Rodríguez, A. (2016, 14 de agosto) En Cartagena afro es solo para pieles negras. El Universal. Recuperado de http://www.eluniversal.com.co/educacion/ en-cartagena-la-catedra-afro-es-solo-para-pieles-negras-232631. 
Simarra, R.; Rodríguez, L.; Muñoz, S.; Hernández, R.; Pérez, T. \& De Ávila, D. (2014). Etnoeducación, Interculturalidad, y Pedagogía Propia. (1 edición), Colombia: editorial Manuel Zapata Olivella. 


\title{
HISTORIAS DE VIDA, RACISMO Y EXCLUSIÓN SOCIAL
}

\author{
Carmen Lago de Fernández ${ }^{1}$ \\ Diana Elvira Lago de Vergara² \\ Liris Munera Cavadías ${ }^{3}$
}

\begin{abstract}
Resumen
Estudiar el RACISMO y una de sus consecuencias la EXCLUSIÓN SOCIAL en una dimensión de Etnoeducación en el Caribe, se constituye en una travesía intelectual de encuentros y desencuentros de las interacciones culturales, mediadas por imaginarios, costumbres, prácticas y convicciones de los pueblos que en su dinámica natural se expresan en comportamientos de exclusión, no contemplados en las normativas políticas de los países, donde la interculturalidad y la integración de los pueblos es el ideal planetario. Este artículo marca rutas desde la literatura del tema que enriquece las investigaciones, y hallazgos de las Historias de Vida, que cada día tributan a un tejido que desde el
\end{abstract}

\footnotetext{
1 Profesora de la Corporación Universitaria Rafael Núñez - CURN, Dra. en Educación, Universidad Complutense de Madrid, España. Investigadora Grupo RUECA-Red Universitaria Evaluación de la Calidad Capítulo Colombia. Coordinadora de la Línea Formación y Desarrollo del Profesorado del Grupo RUECA de la Universidad de Cartagena. Correo: carmenlagodefernandez@yahoo.es

2 Docente de la Universidad de Cartagena. Doctora en Filosofía y Ciencias de la Educación. Universidad Complutense de Madrid. Vicerrectora de Investigación Universidad de Cartagena. Directora del Doctorado en Ciencias de la Educación Universidad de Cartagena - Rudecolombia. Directora del Grupo de Investigacion Rueca - Capítulo Colombia. Correo: dianalago20@ yahoo.es

3 Docente de la Universidad de Cartagena. Mg. en Educación de la Universidad de Cartagena SUE Caribe. Doctora en Ciencias de la Educación Universidad de Cartagena - Rudecolombia. Investigadora y Coordinadora de la Línea Formación y Desarrollo del Profesorado del Grupo RUECA de la Universidad de Cartagena. Coordinadora Académica Licenciatura en Pedagogía Infantil Universidad de Cartagena en convenio con Universidad de Tolima. Correo: lirismunera@hotmail.com
} 